\title{
Qualitative Research in EMDR Therapy: Exploring the Individual Experience of the How and Why
}

\author{
Jamie Marich \\ The Institute for Creative Mindfulness, Warren, Ohio \\ Danielle Dekker \\ Private Practice, Oakland, California \\ Mary Riley \\ The Institute for Creative Mindfulness, Warren, Ohio \\ Adam O’Brien \\ Mutual Arising Counseling, Chatham, New York
}

\begin{abstract}
This narrative literature review examines 12 eye movement desensitization and reprocessing (EMDR) therapy studies published in peer-reviewed journals that implement qualitative methodology other than case studies. Qualitative studies in the EMDR community and in mental health research can get overlooked because they are not perceived to be as scientific as quantitative studies. However, the presence of proper, systematic methodology in qualitative research can reveal another layer of important data about the how and why of EMDR therapy's impact. A variety of study types are reviewed (grounded theory, phenomenology, content and thematic analysis, and several other published forms) that offer evidence-based insight in six major areas of relevance to the EMDR community: the value of the therapeutic relationship and attunement, the role of EMDR therapy preparation and safety measures, the perceived impact of reprocessing phases, and insights for EMDR therapy training and implementation. The authors conclude that it is imperative that clinicians attend to the therapeutic relationship and provide adequate preparation. A discussion about clinical implementation and training EMDR therapists is also included, with suggestions made for advancing qualitative research in EMDR therapy.
\end{abstract}

Keywords: eye movement desensitization and reprocessing (EMDR) therapy; qualitative research; grounded theory; phenomenology; thematic analysis

$\mathbf{E}$ ye movement desensitization and reprocessing (EMDR) therapy is one of the most widely researched therapies for the successful treatment of posttraumatic stress disorder (PTSD), with a growing body of research demonstrating its efficacy in treating other conditions. EMDR is a psychotherapeutic approach based on the idea that maladaptive behaviors, beliefs, and feelings are the result of unprocessed traumatic experiences. The treatment involves a series of standardized procedures rooted in Shapiro's eight-phase protocol, and includes the administration of bilateral dual attention stimulus, with the most widely researched for eye movements, during various phases (World Health Organization, 2013, p. 1). Since founder Dr. Francine Shapiro debuted what developed into the fullness of EMDR therapy in 1989, systematized research has been vital in validating EMDR's treatment efficacy and recognition in treatment guidelines of major clinical organizations internationally (Shapiro, 2018).

For a therapeutic approach like EMDR therapy to be seen as credible and evidence-based, empirical 
research, especially those studies of a randomized controlled or other experimental design, is the field's gold standard. The purpose of this article is not to challenge this reality, but rather to highlight what EMDR therapists may be missing by overlooking qualitative inquiry. This purpose is achieved through a narrative review and examination of 12 major, non-case study articles in the EMDR therapy literature.

EMDR literature reviews typically focus on quantitative studies and often overlook EMDR's qualitative research. Although Shapiro (2018) cited a variety of case studies and case series in the third edition of her text on EMDR therapy, only one qualitative study reviewed by this article appeared as cited material in her text. While case study is a form of qualitative research, there are numerous other approaches to qualitative investigation, including grounded theory, phenomenology, content analysis, critical inquiry, and ethnographic research (Levitt et al., 2018). This article focuses on the qualitative studies that largely get ignored in EMDR therapy literature reviews.

\section{Qualitative Research}

During his keynote address at the 2019 EMDR International Association (EMDRIA) Annual Conference, Dr. Derek Farrell endorsed the importance of qualitative research and encouraged more of it in the EMDR community. He explained that quantitative research is top-down in its orientation, whereas qualitative research is bottom-up. Reisetter et al. (2004), Carey and Stiles (2015), and Whitehouse (in press) contend that qualitative research allows clinicians to feel a stronger connection between research and clinical practice because qualitative research is more likely (than quantitative research) to place value on the individual experience of a person. Some researchers assert that quantitative methods do not optimally reflect clients' experiences in usual care settings (Tucker, Donovan, \& Marlatt, 1999), and that in the case of randomized controlled research especially, controlling for so many variables is not optimally reflective of real-world experiences ( Carey \& Stiles, 2015; Clay, 2010). These contentions are not meant to disparage any form of quantitative inquiry, as many empirical studies within the EMDR literature have been conducted in usual care settings. However, there may be vital nuances missing when the field relies only on one type of research, specifically randomized controlled trials (Carey \& Stiles, 2015). Hill, Thompson, and Williams (1997) contend that richness gets lost when participants are asked to describe their experiences in quantitative metrics only, and that asking open-ended questions is the ideal way to mine for that lost gold.

This tendency to favor the impact of quantitative over qualitative is a noted issue within widescale psychological research and not just in the EMDR community. Because of its open-ended orientation that can focus more on discovery of new phenomenon and less on testing out hypotheses, qualitative research can be seen as non-scientific (Willig, 2013). Qualitative inquiry was vital to the early development of psychological theory, yet as the 19th century progressed, a backlash ensued that continued into the 20th century, based in fear that qualitative inquiry would delegitimize the science of psychology (Levitt et al., 2018). In the last 25 years, qualitative researchers have been working to change this perception and unearthing the areas of knowledge that cannot be discovered by looking at numbers alone (Levitt et al., 2018; Willig, 2013).

Although quantitative methods can prove with numbers how and why something worked for groups of people, qualitative methods are most ideal for revealing the details of how and why something worked for an individual. Qualitative inquiry in psychology assumes that not every facet of the human experience can be quantified. Words and examination of experiences take precedence over numbers in analyzing and making meaning of data (Levitt et al., 2018). In the various traditions of qualitative inquiry, the importance of meaning as an epistemological device (e.g., how and what we know) is emphasized (Willig, 2013).

Debate continues ensues over how the meaning of one's individual experience can be generalized to larger scope of knowledge in the field (De Saint-Georges, 2018), another reason that qualitative research can be devalued. Ercikan and Roth (2016) contend that readers ought to be just as skeptical of undergeneralizing as overgeneralizing the results from qualitative data. Generalizability is fundamentally a quantitative construct and, as an alternative, qualitative research must be evaluated through the lens of transferability - the idea that the findings from one setting can be applied to another. The quality of methodological soundness, specifically the use of thick description (how well the sample population is described) must be used to evaluate the overall potential for transferability (Houghton, Casey, Shaw, \& Murphy, 2013).

Emphasis on methodological quality is important in qualitative research, more so than the traditionally presented concept of rigor in quantitative research that does not necessarily translate as a qualitative construct (Levitt, Motulsky, Wertz, Morrow, \& Ponterotto, 2017). As Korstjens and Moser (2017) suggest, 
the readers will be the ones making the judgment call on quality and ultimately on transferability, yet problems can ensue when readers do not understand qualitative methodology or are reading it with only a quantitative understanding (Levitt et al., 2017, 2018). The importance of methodological quality is a major reason that this narrative review is organized and presented by methodological approach.

\section{Addressing Bias}

In qualitative inquiry of any kind, the practice of epochê (bracketing) is vital. Bracketing asks one to set aside their own worldview in order to gain the experience and the knowledge of another's world (Husserl, 1929/1977). Some argue that scientific observations are always theory-laden or that pure bracketing can never be possible because we can never step outside the contexts that contribute to our bias (Hanson, 1958; Kuhn, 1963/1996), While bracketing in the Husserlian sense is the ideal, it may not be the pragmatic reality, so qualitative researchers must do their best to check and address those biases. Bringing in a team of researchers or writers is another safeguard so that each contributor's work can be checked and assessed.

The primary author acknowledges their potential bias as the author or co-author of three of the 12 articles examined in this literature review. To further keep their biases managed, the first author elected to work on this project with three co-authors, on this project, whose primary tasks were to independently read and offer content analyses of the studies. This decision represents the use of member checking and peer debriefing, two widely recognized skills to minimize bias in qualitative research (Houghton et al., 2013).

\section{Inclusion and Exclusion Criteria}

Qualitative methodologies like grounded theory, phenomenology, and variations on content analyses allow for the examination of more than one case. These forms of inquiry allow investigators to draw thematic conclusions, and they are the focus of this literature review. Unlike Whitehouse's (in press) review of qualitative EMDR studies that focuses solely on client experiences, the authors of this article allowed for the inclusion of several articles that examine providers' perceptions and perspectives. Mixed methods studies containing qualitative components that fit this description are also included.

Single case studies and multiple case studies offered within a single article were excluded from this review.
Although case study methodology technically falls under the purview of qualitative methodology, there are too many to be addressed in a single literature review of this nature. This exclusion does not suggest that single case studies are not important, especially when they highlight the needs of a special population or group. Rather, when qualitative literature is cited in EMDR research or reviews, it is generally the case studies that get the focus. The authors of this article believe it is important to review the other qualitative literature that is more likely to get overlooked. The articles that are of high methodological quality can be of specific interest to clinicians, researchers, and trainers alike for the light they can shed on issues of impact and implementation of EMDR on individuals and systems. A collective decision was made not to include dissertations and masters theses in this particular review that were not later published in peerreviewed journals.

The authors conducted searches using EBSCOHost, psycArticles, and the Francine Shapiro Library using the search terms EMDR and eye movement desensitization and reprocessing in separate combinations with the following search terms: qualitative, qualitative inquiry, qualitative research, qualitative analysis, grounded theory, phenomenology, content analysis, mixed methods. Dissertations, theses, and case studies designs were not searched due to the previously explained exclusion criteria. Multiple case study designs were searched with five returned, yet these were ultimately excluded from the study as they did not incorporate a compare-and-contrast methodology that made them any different from single case designs. Additionally, three studies returned were not included because, although they provided some insight into the lived experiences of people receiving EMDR therapy, no specific methodological system for analyzing the qualitative data was indicated.

Additionally, several literature reviews were found that use some semblance of qualitative methodology to examine studies in the EMDR literature. The authors chose to exclude these from the formal review as they are seen as secondary review sources and not primary research in and of themselves. Two studies that were not in English also could not be included, as resources were not available for translation, and one was excluded since (although it seemed to be a quality design) it appeared in an online newsletter and not a peer-reviewed journal. In total, 12 studies drawn from the peer-reviewed literature meet the inclusion criteria. The article is organized primarily by 
methodological type (e.g., grounded theory, phenomenological design, variations of content analysis, and other published systems).

\section{Grounded Theory}

Glaser and Strauss introduced grounded theory in 1967 as a systematic approach to qualitative research that sought to challenge biases against qualitative research. They posited that theory could be directly extrapolated from the data without the necessity of a prior hypothesis. While sample sizes can vary, they are generally very large compared to other forms of qualitative research since statistical absorption is required in this method (e.g., when additional interviews would not add anything new). According to Thomson (2011), the average sample size of a grounded theory study is 25 . The term grounded implies that the emerging theory ought to be grounded in the data collection and not the researcher's bias. In the EMDR literature there is only one grounded theory study (Ricci \& Clayton, 2008), and one study that used an aspect of grounded theory, constant comparison (Marsden, Lovell, Blore, Ali, \& Delgadillo, 2017).

The first study is the qualitative arm of an earlier parent study that Ricci and Clayton conducted with Dr. Shapiro on the use of EMDR therapy in treating sex offenders. The parent study found that adding EMDR to standard cognitive-behavioral therapy (CBT) for relapse prevention (CBT-RP) treatment resulted in statistically significant pre-post improvement on all six subscales of the Sex Offender Treatment Rating Scale (Ricci, Clayton, \& Shapiro, 2006). Their qualitative data consisted of interviews and treatment transcripts with the original study's sample of 10 white males. While all participants reported a history of childhood sexual abuse, specific PTSD diagnoses were not indicated. They provided a copy of their self-developed interview in the appendix, specifically designed using language participants could understand, relevant to the research aims. In the sample, an average of six EMDR therapy sessions were administered to each participant specifically addressing targets related to emergent blocks in the CBT-RP work. While the sample size of 10 is generally considered small for grounded theory (and they did not make any notes on accounting for statistical absorption specifically), all of the other elements of the quality (specifically the constant comparison technique) and checks for biases in grounded theory work appear to be present.
Themes that emerged from the data analysis include subjects' recognition of their distorted beliefs (and contribution factors); increased accountability, empathy, and participation in group therapy; clarification of thoughts; raised consciousness as a selfmanagement tool; and increases in self-esteem and emotional regulation/management. The themes and subthemes align with CBT-RT objectives, demonstrating that EMDR therapy served to strengthen the desired outcomes. Their discussion recognizes the limitations of the study while also acknowledging that the findings support a contention in the sex offender literature that addition of a trauma resolution therapy could enhance the efficacy of the CBT-RT.

Like the Ricci and Clayton study, the other work in this category from Marsden et al. (2017) originated in a parent study, a randomized controlled study of 55 participants that found no significant difference between CBT and EMDR for treatment of obsessive compulsive disorder. The qualitative component consisted of taking 24 willing participants through an existing semistandardized interview to make comparisons of how members of the two groups perceived their treatment. The interviews were coded and analyzed using the constant comparison (meanings of all categories are compared and contrasted) and presented in three categories: common threads between EMDR and CBT groups, specific experiences of CBT patients, and specific experiences of EMDR patients. The common findings were numerous (with six-11 themes in each of these categories) and generally refer to the common factors that are likely present in all psychotherapy. The most pronounced common thread highlighted in the discussion was that the nature of the patient's relationship with their therapist was an important part of their perceived progress. Supportive and non-judgmental are the two major adjectives used to describe effective therapists in both groups. Participants in both groups describe powerful experiences in turning a corner after a breakthrough.

Specific to the EMDR group, participants reflected positively and favorably on the use of preparation techniques like the "Safe Place" and grounding. However, many participants in the EMDR group (much more so in the CBT group) had difficulty describing the rationale of the treatment and struggling with certain aspects of the method (e.g., being able to "bring something up" with adequate charge when asked). According to the investigators, this lack of clear understanding possibly contributed to treatment drop-out in some cases. 


\section{Phenomenological Design}

In this category a strong EMDR advocate, Marich, coauthor of this article, was involved in all three phenomenological studies. While this may be viewed as a potential bias, she used one of the most credible (Levitt et al., 2018) methods for reading data phenomenologically for her solo work: Giorgi's $(1997,2003)$ Descriptive Phenomenological Psychological Method. This practical method, rooted in the work of Edmund Husserl, studies the phenomenon of lived experience. Marich's (2010) major study on the use of EMDR therapy in addiction continuing care with women is the first in this section, followed by a separate study flowing from the same data collection focused specifically on qualities of a good EMDR therapist.

Although Marich's (2010) original study consisted of only women, it is one of the most racially and ethnically diverse in the EMDR literature, with half of the 10 participants identifying as persons of color. Marich used Grant McCracken's (1988) The Long Interview as the guide for her semi-standardized interview instrument. She tailored some of the questions to be specific to EMDR therapy and the research questions which were specifically designed to investigate how EMDR worked in the addiction continuing care process. A specific PTSD diagnosis was not required of participants to take part in the study, although every participant reported a history of trauma in both childhood and adulthood. All of the participants met criteria for a primary substance use disorder. Marich worked as the interviewer and primary coder on the project. Marich's dissertation advisor provided checks on her coding work throughout the process.

The parent study concluded that there is a place for EMDR therapy in the larger scope of addiction care. Four key themes offer insight into the how: (a) safety precautions being in place (which includes the therapeutic relationship, safety plan for in between sessions, adequate preparation), (b) having their initial skepticism about EMDR therapy addressed, (c) accessing the emotional core wounds, $(d)$ the role of EMDR in impacting lifestyle change, and the usefulness of combining factors in successful treatment (e.g., EMDR being used alongside programs like 12-step recovery, and other groups at the facility). This phenomenological design of usual care treatment allowed for insight into various levels of engagement into EMDR therapy. Meeting the needs of clients on a case-by-case basis, as determined by clinician judgment and client collaboration, reflected the ethic of the treatment center and their use of EMDR therapy.
There was such abundant data presented by all of the participants on the impact of the therapeutic relationship in their EMDR therapy experiences that Marich (2012) conducted a separate study focusing on just this material through the lens of the Giorgi method. The specific qualities of what patients perceived to be a good EMDR therapist included therapist personality, an ability to empower clients, flexibility, intuition, a sense of ease and comfort in working with trauma, and a commitment to the small measures of caring that clients identify as helping them feel safer. Insight into the importance of clinician judgment and knowing how and when to bring in EMDR therapy interventions into a multifaceted treatment experience was also gleaned from this reading of the data. More specific vignettes and examples appeared in the article to illuminate these descriptions. Two of these vignettes described negative client experiences with their EMDR therapists the first time they attempted it at the center with a therapist they perceived to be unskilled and ill-prepared to work with trauma. These clients described their ineffective EMDR therapist as rigid, scripted, anxious, unclear, and not able to handle trauma; as soon as they were switched to another therapist at the center who met the positive qualities, their reported experience changed. Allowing for negative case analysis like this and not filtering out negative experiences with a phenomenon being investigated is a vital credibility check in the Giorgi method (2003) and in qualitative research as a whole (Morse, Barret, Mayan, Olson, \& Spiers, 2000).

In a similar-style study, Wise (the primary investigator) interviewed patients of other EMDR therapists, with Marich serving as the advisor who conducted the coding checks. Creswell's (2013) practical method based on the work of Moustakas (1994), another major system for reading, coding, and analyzing phenomenological data, was incorporated for this study. The aim of this particular phenomenological design was to investigate the lived experience of clients with a PTSD diagnosis and a self-described addiction problem who experienced EMDR therapy in an outpatient or private practice setting. Wise asked questions to determine perceived differences in experience between the standard EMDR therapy protocol and the specialty protocols published in the EMDR community to work with addictions. Wise recruited participants through therapists who are known to specialize in the concurrent treatment of trauma and addiction. They verified which of the specialty protocols they used in treatment, as well as diagnoses and impressions about addictive behavior (five identified issues 
with substances only, two identified issues with behaviors, and two identified issues with both substances and behaviors).

All participants reported positive outcomes from the combined EMDR approaches, with four major themes emerging: (a) recognition of their trauma and addictions as related (resulting in changes to thoughts and behaviors), (b) recognition of remission of traumarelated and substance use disorder-related symptoms with EMDR therapy reported to be effective whether the traumatic symptoms were treated before or after the addictive symptoms, (c) realization that integrated treatments (including other supportive services) were optimum for their ongoing recovery, and (d) report that the relationship with the therapist was integral to the overall success of treatment. Working on traumatic memories did not increase client desire to use, addressing a common fear that EMDR therapy will "cause" a client to relapse. From the perspective of the clients, the relationship was identified as a factor in helping their therapists make decisions about when to use which protocols and at what time.

\section{Content/Thematic Analysis}

In the broadest sense, content analysis (of which thematic analysis is a component) refers to any technique of qualitative reading that allows a researcher to make inferences by systematically and objectively identifying the characteristics of a message (Holsti, 1968). A host of material can be studied through content analysis, including photography, field observations, therapeutic service notes, and interviews. A criterion of selection must be chosen and rigorously applied to avoid the problem of only the researcher's hypothesis being supported. These selection criteria can be a pre-established set of criteria (i.e., this is what we're looking for and how we're looking for it) or a predetermined system like many that will follow in this section and the next section. The first two studies identified the thematic analysis guide of Braun and Clark (2006) to read their data, with the third using that of Saldaña (2015).

Cottner, Meysner, and Lee (2017) first conducted a randomized controlled parent study on the use of CBT versus EMDR in the treatment of individuals who reported that they needed help with grief, with no significant difference found between the two treatments. Independent readers may view this lack of an overtly favorable result for EMDR therapy in the parent study as enhancing the team's credibility and objectivity for the qualitative arm. Cottner et al. recognized that asking the participants about how they experienced the therapy may provide a needed dimension, and 18 of the original study participants agreed to a well-constructed semi-standardized interview. No specific clinical diagnosis was required to participate, simply an identification of difficulty coping with grief following the death of a loved one.

Like the study conducted by Marden's team, Cotter et al. laid out their findings by describing the similar themes between both CBT and EMDR therapy (increased insight, positive shift in emotion, shift in mental relationship to the deceased, increased selfconfidence, and increased activity levels). The participants in the CBT group felt they were more equipped with tools and skills to handle emotions in life (not reported by any of the EMDR participants) and were equipped with an ability to move forward. In the EMDR group, a greater sense of distancing from the memories was reported (not indicated by any participants in the CBT group). The primary investigators were not the ones who conducted the interviews, and they indicated having supervision in their coding process. This particular study demonstrates the value of a qualitative method to reach deeper levels of nuance, explaining differences in client experience after the quantitative parent study showed similar outcomes.

Hurn and Barron (2018) also called upon Braun and Clark's thematic analysis method to read their data in their investigation of the Integrative Group Treatment Protocol (IGTP) in a psychosocial program for refugee children. Description suggests that the researchers performed the coding and others provided the therapy. This inquiry examined the use of IGTP with a group of eight Arabic-speaking children (five boys and three girls) in a psychosocial context where interpreters were used. The children in the study were described as having experienced emotional distress, yet no specific diagnoses were indicated.

The researchers reported taking subjective units of disturbance (SUD) ratings and presented the results. They referenced Session Rating Scales and Outcome Ratings Scales, although these were presented in a more qualitative fashion as they allowed for uses of drawings and prose descriptions. The most robust qualitative content for analysis came from extrapolating the themes from interviewing the two therapists and four interpreters about their experience. The therapists' statements were grouped into the themes that describe their observations about this particular delivery system of IGTP. The non-pathological nature of the program, engaging children through social/cultural education, the strength of the interpreters, the 
role of music as a cultural facilitator, and the ability of groups to provide further assessment for individual work within the family were all identified.

Some challenges were also identified by the therapists, including their assessment that two of the children may not have been a good fit for group work. The interpreters also offered valuable insight on what worked and what may need improvement. For instance, translating the phrase mental health as psychological health may be more appropriate for Arabic speakers. In sum, this article is a valuable read for EMDR therapists who work with groups and/or those who work through interpreters for insight about implementation. The trauma aspect of the treatment (EMDR) was rated the least enjoyable but most effective by clients, so the additional psychosocial elements of the group (rated as highly enjoyable) may be the key to engaging children.

The final article in this section is also valuable for EMDR therapists wanting to learn more about cultural competence and related connections. DiNardo and Marotta-Walters (2019) presented a cultural vignette to 56 EMDR-trained therapists recruited through major organization Listservs. Each participant was asked to read an EMDR case vignette and respond to six questions presented about the vignette that focused on culture. Using a method of basic interpretative and discourse content analysis guided by Saldaña (2015), six major themes emerged with a long list of subthemes and explanatory statements that are too extensive to cover in this review. Evaluation of how the largely White-identified providers conceptualized EMDR therapy and how they discussed EMDR with their clients predominates the discussion component of this article, with the researchers raising concerns about the lack of therapist awareness in many areas (e.g., therapists' word choice, their belief that the EMDR standard protocol is so universally applicable that culture does not need to be addressed). A call to action is made for EMDR therapy training programs to more specifically address culture, with many of the participants identifying that no mention was made of cultural attunement in their basic trainings. Clinical implications arrived at by the authors include increasing space for cultural discourse in EMDR therapy trainings, adapting metaphors for describing the EMDR therapy process to people of cultural backgrounds different than that of the trainer, and making neurobiological explanations of EMDR and trauma therapy more accessible.

\section{Other Published Systems for Qualitative Analysis}

This section explores four well-designed qualitative studies that each make use of a reputable, published system of analyzing qualitative data. While all of the systems have their own methods of how to read data, they have the same essential goal of providing readers with a system for coding and interpreting data. There are similarities to content and thematic analyses in each of these systems, although each impart a unique spin developed by the authors of each system.

The first well-known, study in this section is another follow-up to a parent study that tested EMDR against comparison conditions with survivors of sexual abuse (Edmond, Sloan, \& McCarty, 2004). This is the only study from this narrative review that Shapiro (2018) cited in the third edition of her textbook on EMDR therapy. The parent study, a randomized controlled investigation by Edmond, Rubin, and Wambach (1999), found that both EMDR and eclectic therapy showed statistically significant reduction in trauma symptoms compared to the waitlist control condition; no significant differences were noted between EMDR therapy and eclectic therapy.

A desire to investigate the true impact of either therapy seemed to motivate the qualitative component (Edmond et al., 2004), which consisted of interviewing 38 of the original 59 women in the study (predominantly white) using McCracken's (1988) The Long Interview. This recognized system of obtaining qualitative data was used to inform the questions (presented in the article) and provide a structure for analyzing the data. The overall finding was that women who experienced the eclectic therapy placed much more importance on the value of the therapeutic relationship and connection to the therapist than those who received EMDR therapy.

The experiences shared by the EMDR therapy group seemed to reflect those qualities of change that are more indicative of trauma resolution. Although Edmond et al. (2004) noted in their discussion that the finding does not negate the importance of the therapeutic relationship, suggesting that the relational elements are already woven into the foundation of EMDR, the results indicated that the relationship was not of primary value to the EMDR clients in this study. The qualitative method in the study was able to unearth that the two treatment groups had different experiences in treatment and different views of 
the nature of the change even though the quantitative results showed comparative levels of high satisfaction in treatment outcome. The authors referred to the EMDR clients' theme of trauma resolution as the "holy grail of desired clinical outcomes" (p. 269). That would have been lost had the qualitative investigation not been done, and thus demonstrates the clinical merit of combining the two approaches to inquiry.

The other three studies in this section largely focus on issues of professional training in EMDR and dissemination of the method, so the experiences of therapists were examined. DiGiorgio, Arnkoff, Glass, Lyhus, and Walter (2004) conducted interviews with three EMDR therapists previously trained in other orientations-psychodynamic, humanistic, and cognitive-behavioral-about their implementation of EMDR therapy into the clinical setting. Using the Consensual Qualitative Research (CQR) Model (Hill et al., 1997) to analyze the data, the chief finding is that all therapists interviewed acknowledged modifying the standard EMDR therapy protocol in which they were trained to emphasis the elements of their original approach to psychotherapy. Numerous points of discussion are made about psychotherapy integration and the person of EMDR therapist as a critical vehicle in the method. EMDR therapy can be haphazardly implemented without any checks and balances, yet flexibility and integration with other therapies can also strengthen its potential for targeting a range of clinical issues.

Cook, Biyanova, and Coyne (2009) interviewed sets of providers at two sites within the Veterans Administration (VA) system -10 providers at a site where EMDR was adopted and 19 at a site where it was not. Using Yin's (2003) Explanation Building Method to conduct the semi-standardized interviews and analyze the results, the researchers concluded that at the site where EMDR was adopted, critical selling points for EMDR included the presence of a highly regarded "EMDR champion" on site, observable impact on patients, and personally experiencing its effects during training sessions. A factor that that helped in dissemination was the availability of yearly on-site EMDR therapy training at no charge to eligible clinicians. Moreover, EMDR's compatibility with existing schools of psychotherapy further allowed EMDR to be accepted by the site's culture and operational standards, an endorsement for integration that parallels what DiGiorgio et al. (2004) presented. For the site where EMDR was not adopted, the reasons included a sense that EMDR was not theoretically coherent and seemed to be more about business than science. Even when presented with empirical evidence to the contrary, the bizarre or different nature of EMDR therapy and difficulty with understanding how it works seemed to be a barrier.

Farrell, Keenan, Knibbs, and Hicks (2013) investigated six individuals trained in Pakistan through EMDR Europe HAP. The investigators chose the comprehensive Q-methodology (Ellingsen, Storkson, \& Stephens, 2010) for investigating and analyzing the experiences of the participants, citing its qualitative nature combined with implementational rigor usually seen in quantitative approaches. Through card sorting of experience statements, the main feature of Q-methodology, experiences about EMDR practice, cultural implementation within Pakistan, research and development, and general experiences were investigated. Use of such a systematized procedure could also be viewed as necessary for objectivity since the study was conducted by known advocates of EMDR therapy. The participants reflected favorably on the theoretical basis for EMDR therapy and that it can be applied to more than just PTSD. The practicum components and supervision/consultation components of the training were also identified as vital.

The importance of attending to cultural factors with negative and positive cognitions when explaining EMDR therapy to a client is emphasized. Themes also emerged related to EMDR therapy's compatibility with Eastern approaches to psychotherapy and healing in general, although it is important to take cultural elements around topics such as touch, cultural attire, and gender aspects into account. There are also insights derived on reconceptualizing EMDR therapy as a method that could be effectively used with a wider variety of populations than PTSD as dysfunctional memory networks are ubiquitous and not distinct to PTSD. Many other specific pieces of insight from this study would be of particular interest to EMDR therapy trainers committed to culturally attuning EMDR therapy training.

\section{Discussion}

This article has the distinction of assembling a variety of qualitative methodologies other than case study to explore individual experiences with EMDR therapy. These 12 studies represent the use of quality in methodology, a term that is preferred to the more quantitative construct of rigor; quality is an important component in assessing the credibility and transferability of qualitative research (Houghton et al., 2013). 
See Table 1 for a full presentation of the methodological features, the perspectives examined, and the key findings from each study. All 12 studies seemed to allow for this sense of spaciousness to see what would be revealed instead of having an agenda about presenting EMDR therapy in a positive light. Allowing negative material to be analyzed and presented is vital for qualitative research to remain credible (Morse et al., 2000), especially when qualitative research can be viewed as non-scientific and inherently biased (Levitt et al., 2018).

With case studies being the most common presentation of qualitative research in the EMDR community (especially in Shapiro's text), featuring these studies is a major step forward in exposing EMDR therapists, researchers, and trainers to the possibilities of what qualitative research can reveal. This narrative review also has the distinction of featuring many different client populations and settings examined for perspectives, insights, and experiences. Perspectives of providers and ancillary support figures like translators (see Hurn \& Barron, 2018) are also woven into this literature review. Several key themes emerge that will be highlighted in the sections that follow.

\section{The Therapeutic Relationship and Attunement}

This narrative review endorses the value of the therapeutic relationship (Dworkin, 2005) and attunement in the delivery of EMDR therapy. Clinical participants in four studies specifically referenced the importance of therapist relationship in their perceived success with EMDR therapy (Marich, 2010; Marich, 2012; Marsden et al., 2017; Wise $\&$ Marich, 2016). These experiences support the contention made by the recent Whitehouse (in press) qualitative review of client-only experiences that the therapeutic relationship is paramount. A different experience was reported by Edmond et al. (2004). In that study, clients did not view the therapeutic relationship as related to the positive outcomes they experienced in treatment. However, the authors acknowledge that a great deal of relational elements are inherently woven into EMDR therapy, and they do not dismiss the therapeutic relationship as unimportant.

In addition to alliance, the importance of attunement-best represented by a willingness to adapt in order to meet clients at their level of readiness-emerges thematically. Wise and Marich (2016) emphasized the importance of clinical discretion in decision-making. Qualities like flexibility, intuition, ease in working with trauma, and attending to small measures of caring were also noted by clinical participants in another study from the same methodological grouping (Marich, 2012). The identified importance (DiGiorgio et al., 2004; DiNardo \& Marotta-Walters, 2019; Farrell et al., 2013; Hurn \& Barron, 2018) of cultural awareness and willingness to adapt to individual needs are examples of attunement.

\section{EMDR Therapy Preparation and Safety Measures}

A recurring theme in several studies was the importance of safety, preparation, and orientation, all elements of EMDR therapy Phase 2 (Shapiro, 2018). Clients expressed appreciation for their direct experiences of these elements in four of the clinical studies (Marich, 2010; Marich 2012; Marsden et al., 2017; Wise \& Marich, 2016). These findings may shed some important light on an often contested topic: How much preparation is adequate? This is an area of ongoing debate in the field of trauma-focused therapy (Van Toorenburg et al., 2020). While Shapiro (2018) has long contended that preparation is not processing, her third edition robustly welcomes ancillary and proactive measures, especially with more complicated cases. On the other hand, De Jongh et al. (2016) challenged long-standing beliefs about the imperative of stabilization in the treatment of complex trauma, suggesting that emotional regulation may not be possible until trauma-focused treatment commences. As this review emphasizes the importance of the lived client experience as a vital source of data collection, it may be most clinically sound to attend to the needs of a client on an individualized basis.

\section{The Experienced Impact of Reprocessing Phases}

While qualitative methodology cannot shed definitive light on mechanism of action or how something works overall, participants throughout the studies offered reports based in their own experiences about how EMDR therapy seemed to help them. Clinical participants in two of the articles (Cotter et al., 2017; Edmond et al., 2004) reported a greater sense of resolution and distancing from the memory as a result of EMDR therapy. Other experiences reported include EMDR's role in helping to facilitate a breakthrough (Marsden et al., 2017), EMDR's role in helping to access and process core emotional issues (Marich, 2010), and EMDR's role in helping to engender positive lifestyle change (Marich, 2010; Wise $\&$ Marich, 2016). Ricci and Clayton (2008), whose use of classical grounded theory makes them pioneers in the field 


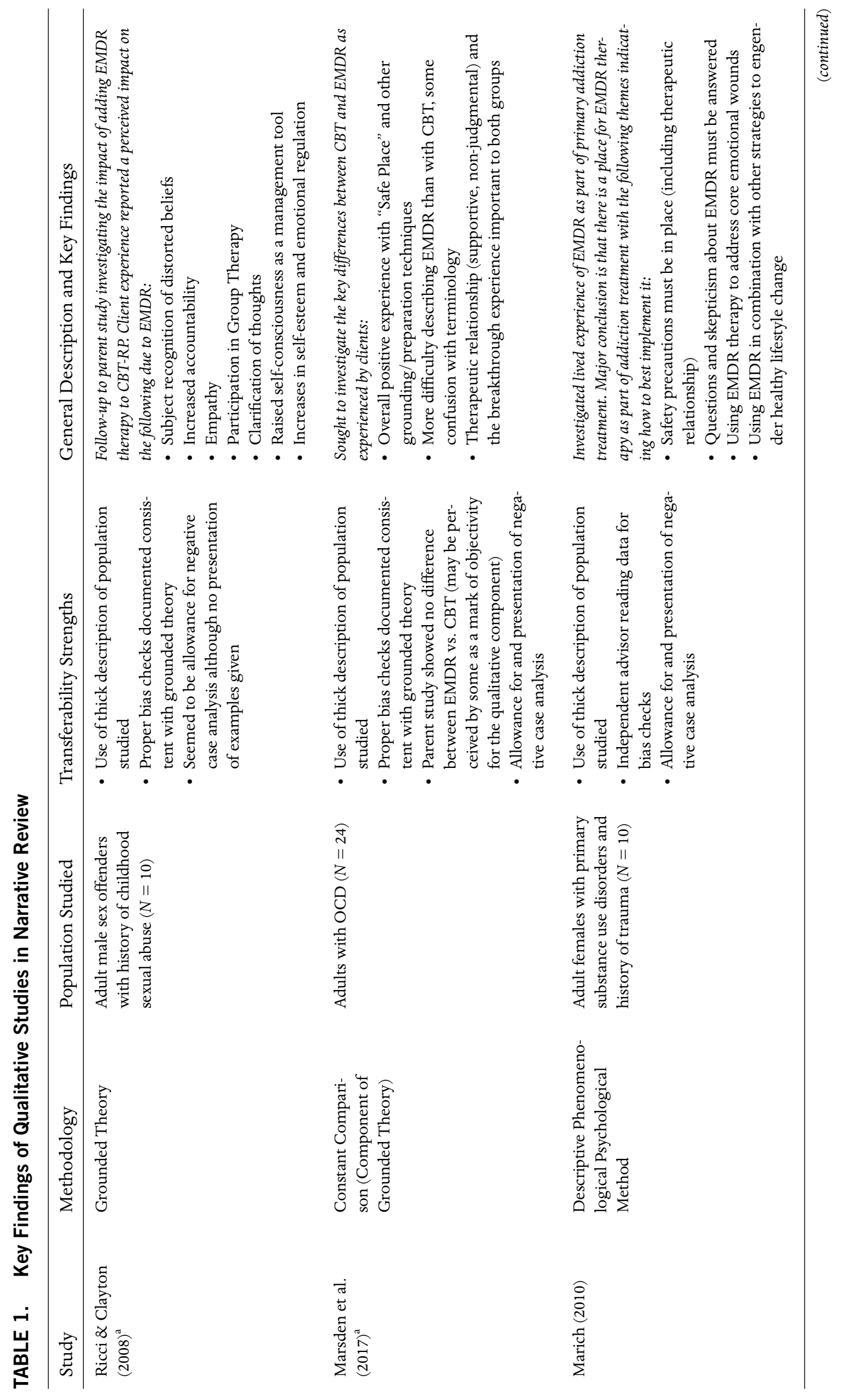




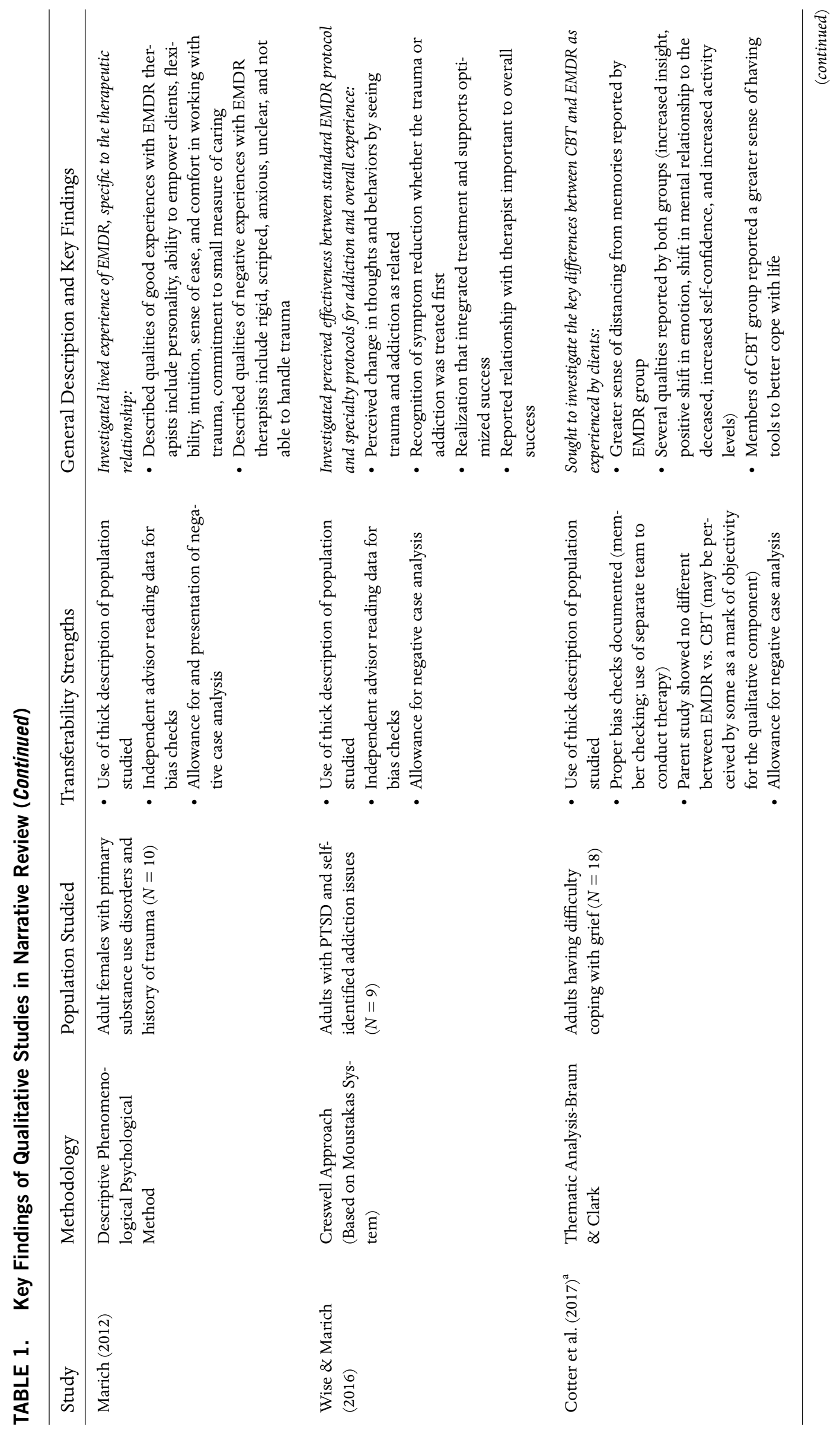




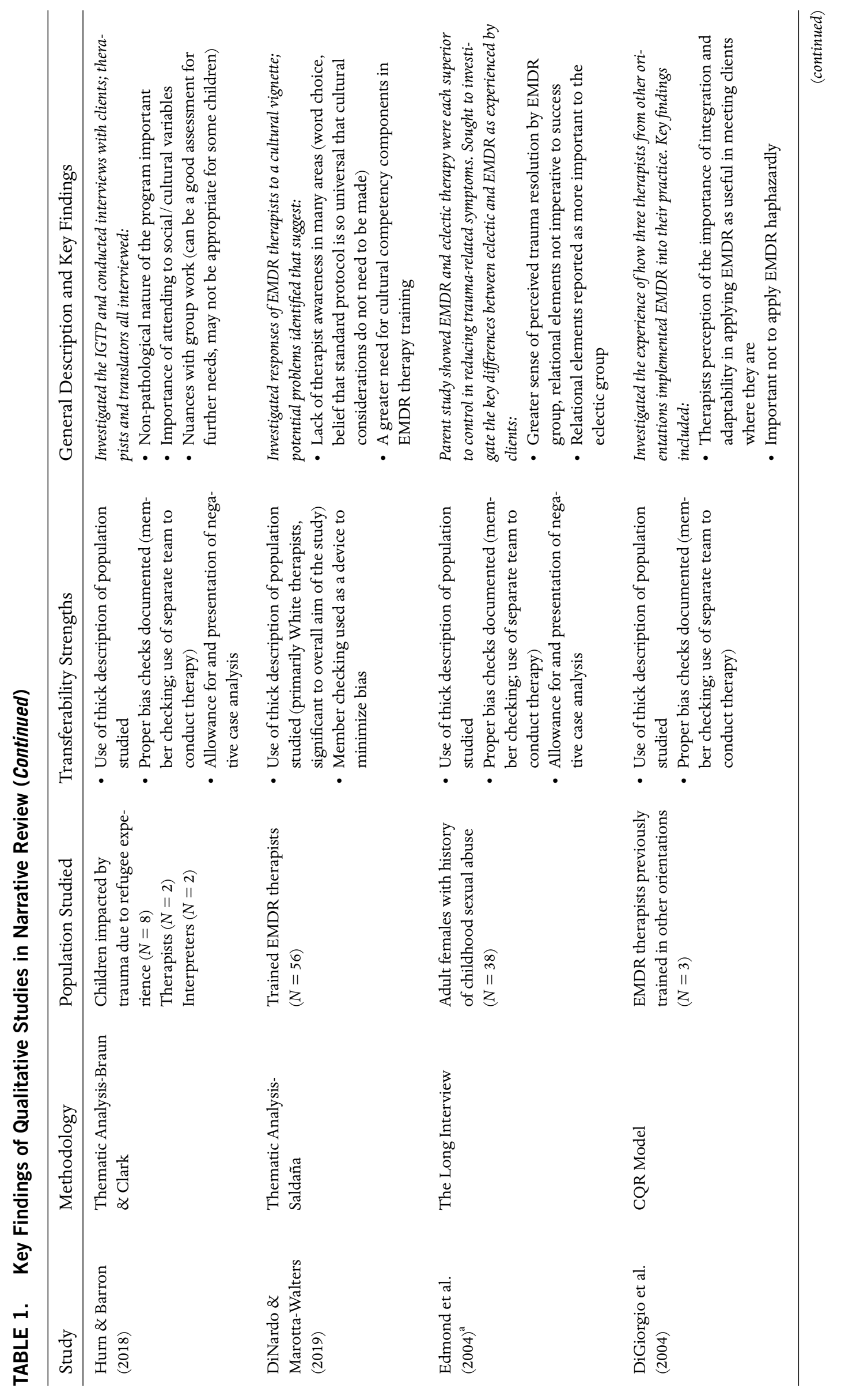




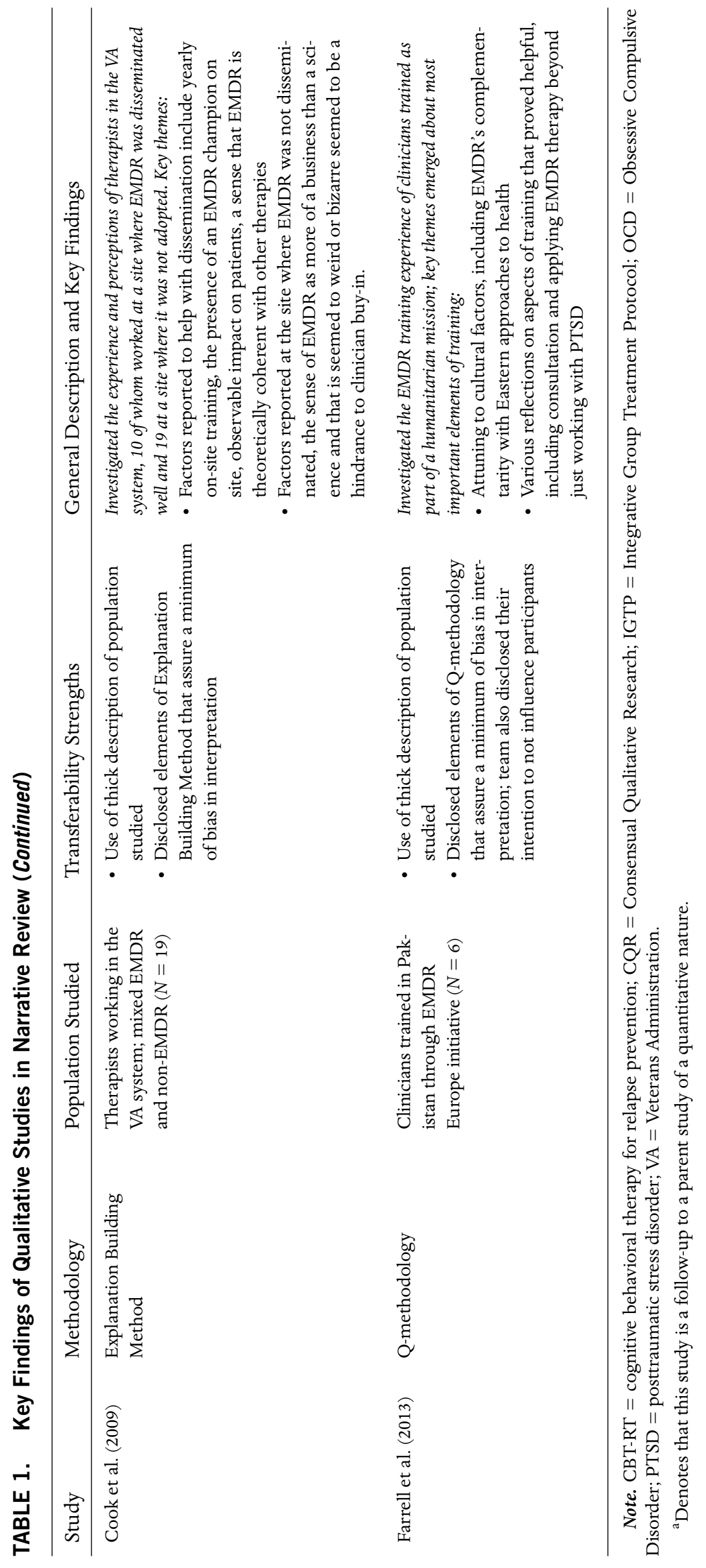


of qualitative EMDR research, reported a variety of positive experiences from clients due to their EMDR experiences. EMDR's perceived ability to help them more fully engage in CBT-RT treatment groups was a major noted benefit, as hoped for by the clinical team. The key factors for change brought about by the EMDR, as perceived by the participants, included recognition of distorted beliefs and clarification of thoughts.

\section{Insights for EMDR Therapy Training}

Specific research and outcomes on training and development of EMDR therapists is limited. The five studies that explored the experiences of therapists who received EMDR training and went on to implement the approach are thus especially valuable in the EMDR literature. Attuning to culture, or having an understanding of the background of people being trained and the people they are going on to serve, is imperatively highlighted in the conclusions of each study that investigated training. In the Cook et al. (2009) study, the accessibility and affordability of training emerged as an important consideration.

\section{Cultural Adaptations}

In both training and clinical work in non-Eurocentric humanitarian settings, a necessity is articulated for culturally attuned application of EMDR therapy (Farrell et al., 2013; Hurn \& Barron, 2018). However, this need for cultural attunement and further modifications based on culture is not just an issue in humanitarian settings. For instance, DiNardo and Marotta-Walters (2019) expressed serious concerns in their conclusions that the EMDR therapists they investigated, primarily white North Americans, believed that cultural modifications were not needed and that the standard EMDR protocol is sufficiently universal across cultures. They further concluded that standard trainings in EMDR therapy must include more of a crosscultural component, specifically on the nuance of language, if EMDR trainings are going to serve a more diverse world. Three studies in this review (DiGiorgio et al., 2004; Farrell et al., 2013; Hurn \& Barron, 2018) suggested that it is viable to implement EMDR therapy alongside other modalities to more effectively modify and meet people where they are at in the change process.
Providing an Adequate Rationale for EMDR Therapy

Di Nardo and Marotta-Walters reported that therapists have difficulty explaining EMDR to their clients. Negative case analysis in two studies revealed that some clients were confused by how EMDR therapy worked, which included problems with terminology and clarity that impacted retention in treatment for some (Marsden et al., 2017), and for others, their trust in EMDR therapy (Marich, 2012). Cook et al. (2009) investigated and compared two VA sites, one that adopted EMDR therapy and one that did not. Refer to Table 1 for the specific breakdown of findings. A general contention can be made that having an EMDR advocate on site, especially one who can explain EMDR clearly and as scientifically as possible, is essential. EMDR's bizarreness and appearance as a business more than science were a hindrance for many. Considering such comments can give trainers and policymakers pause as they consider how to best present EMDR to clinicians eligible for training. The EMDR community is advised to develop better and simpler rationales for EMDR's implementation that can be easily understood by the general public.

\section{Future Directions}

When their study was published in 2004, Edmond et al. articulated there was insufficient qualitative investigation within EMDR literature. Their study is one of the oldest in this narrative review, and it is encouraging to see that more non-case study articles are now in the peer-reviewed literature. Many studies in this review occurred as follow-ups to a quantitative, parent study. These qualitative components unearth potent clinical insight on the client's experience. EMDR therapy researchers with more of a quantitative approach to research may consider adding a qualitative component, even if it is small, to enrich their investigation. While the qualitative components in these parent studies followed the quantitative ones, in the general field of psychology, qualitative inquiry usually comes first to provide a foundation for further exploration (Levitt et al., 2018). It would be interesting to see more EMDR research teams adopt this bottomup approach, or to see researchers featured in this review, who set a solid qualitative foundation with 


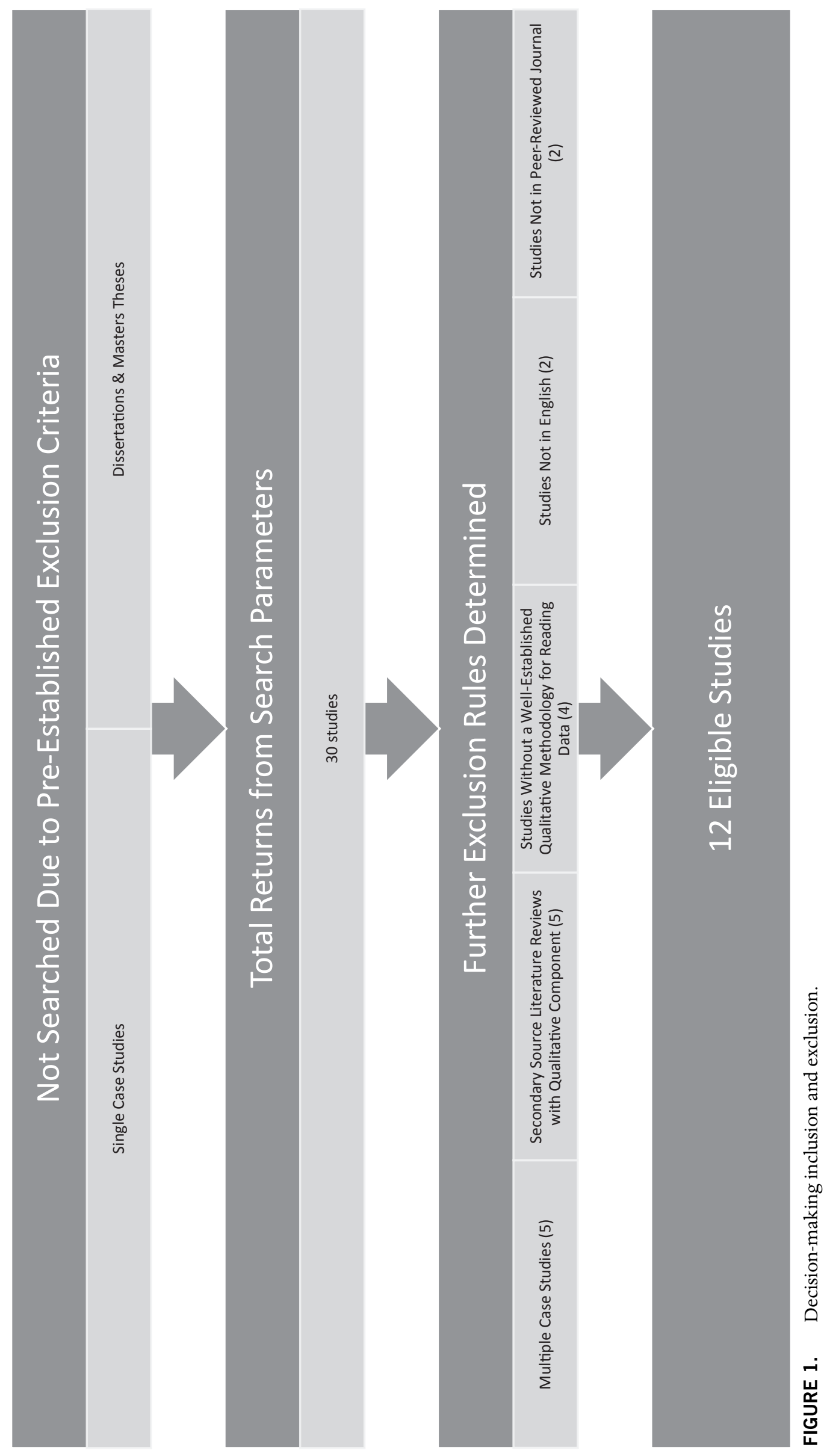


their inquiry, go on to investigate their phenomenon in a quantitative realm.

There are a variety of experiences and perspectives that are still out there to be mined. Qualitative inquiry can be used to help investigate the perceived role of the therapist in different styles and applications of EMDR therapy. For instance, lived experiences with the different forms of delivery in bilateral stimulation can be explored, or investigating how and what style of preparation is needed in ensuring for adequate readiness to manage affect in the reprocessing phases of EMDR therapy. While qualitative research is often looked down upon for having small sample sizes, consider that in each experience and perspective may lie a new piece of the puzzle for further understanding the impact of EMDR therapy in transforming the legacy of unhealed trauma. All three clinical authors of this study reported that going through these articles directly translated to giving them new clinical insight or affirming an existing relational practice they already used. The lead author immediately noticed a translation to their EMDR training style and feeling more validated in their existing commitment to highlight both relational and culturally attuned elements of client experience instead of pure focus on technique (Figure 1).

\section{References}

Braun, V., \& Clarke, V. (2006). Using thematic analysis in psychology. Qualitative Research in Psychology, 3(2), 77- 101.

Carey, T., \& Stiles, W. (2015). Some problems with randomized controlled studies and some viable alternatives. Clinical Psychology \& Psychotherapy, 23(1), 87-95.

Clay, R. (2010). More than one way to measure. Monitor on Psychology, 41(8), 52.

Creswell, J. (2013). Qualitative inquiry \& research design (3rd ed.). Los Angeles, CA: Sage.

Cook, J. M., Biyanova, T., \& Coyne, J. (2009). Comparative case study of diffusion of eye movement desensitization and reprocessing in two clinical settings: Empirically supported treatment status is not enough. Professional Psychology: Research and Practice, 40(5), 518-524.

Cotter, P., Meysner, L., \& Lee, C. W. (2017). Participant experiences of eye movement desensitization and reprocessing v. cognitive behavioral therapy for grief: Similarities and differences. European Journal of Psychotraumatology, 8, 1-10.

Dworkin, M. (2005). EMDR and the relational imperative: The therapeutic relationship in EMDR treatment. New York: Brunner-Routledge.
De Jongh, A., Resick, P., Zoellner, L., van Minnen, L., van Minnen, A., Lee, C. , ...Bicanic, I. (2016). Critical analysis of the current treatment guidelines for complex PTSD in adults. Depression and Anxiety, 00, 1-11.

De Saint-Georges, I. (2018). Generalizing from case studies: A commentary. Integrative Psychological and Behavioral Sciences, 52, 94-103.

DiGiorgio, K. E., Arnkoff, D., Glass, C. R., Lyhus, K. E., \& Walter, R. C. (2004). EMDR and theoretical orientation: How therapists integrate eye movement desensitization and reprocessing into their approach to psychotherapy. Journal of Psychotherapy Integration, 14(3), 227-252.

DiNardo, J., \& Marotta-Walters, S. (2019). Cultural themes and discourse in EMDR therapy. Journal of EMDR Practice and Research, 13(2), 111-123.

Edmond, T., Rubin, A., \& Wambach, K. (1999). The effectiveness of EMDR with adult female survivors of childhood sexual abuse. Social Work Research, 23, 103-116.

Edmond, T., Sloan, L., \& McCarty, D. (2004). Sexual abuse survivors' perceptions of the effectiveness of EMDR and eclectic therapy. Research on Social Work Practice, 14(4), 259-272.

Ellingsen, I. T., Størksen, I., \& Stephens, P. (2010). Q methodology in social work research. International Journal of Social Research Methodology, 13(5), 396-409.

Ercikan, K., \& Roth, W. M. (2016). Qualitative and quantitative evidence in health: The critics' view. In K. Olson, R. Young, \& I. Schultz (Eds.), Handbook of qualitative health research for evidence-based practice. Handbooks in health, work, and disability (Vol. 4). New York, NY: Springer Publishing.

Farrell, D., Keenan, P., Knibbs, L., \& Hicks, C. (2013). A Qmethodology evaluation of an EMDR Europe HAP facilitators training in Pakistan. Journal of EMDR Practice and Research, 7(4), 174-185.

Giorgi, A. (1997). The theory, practice, and evaluation of phenomenological methods as a qualitative research procedure. Journal of Phenomenological Psychology, 28(2), 235-281.

Giorgi, A. P., \& Giorgi, B. M. (2003). The descriptive phenomenological psychological method. In P. M. Camic, J. E. Rhodes, \& L. Yardley (Eds.), Qualitative research in psychology: Expanding perspectives in methodology and design (pp. 243-273). Washington, DC: The American Psychological Association.

Glaser, B., \& Strauss, A. (1967). The discovery of grounded theory: Strategies for qualitative research.. Chicago, IL: Aldine.

Hanson, N. R. (1958). Patterns of discovery. Cambridge: Cambridge University Press.

Hill, C. E., Thompson, B. J., \& Williams, E. N. (1997). A guide to conducting consensual qualitative research.. The Counselling Psychologist, 25, 517-572.

Holsti, O. R. (1968). Content analysis. In G. Lindzey \& F. Aaronson (Eds.), The handbook of social psychology. Reading, MA: Addison-Wesley. 
Houghton, C., Casey, D., Shaw, D., \& Murphy, K. (2013). Rigour in qualitative case-study research. Nurse Researchers, 20(4), 12-17.

Hurn, R., \& Barron, I. (2018). The EMDR integrative group treatment protocol in a psychosocial program for refugee children: A qualitative pilot study. Journal of EMDR Practice and Research, 12(4), 208-223.

Husserl, E. (1977). Cartesian meditations: An introduction to phenomenology (D. Cairns, Trans.). New York, NY: Springer Publishing. (Original work published 1929)

Korstjens, I., \& Moser, A. (2017). Series: Practical guidance to qualitiave research. Part 4: Trustworthiness and publishing. European Journal of General Practice, 24(1), 120 124.

Kuhn, T. S. (1996). The structure of scientific revolutions. Chicago, IL: University of Chicago Press. (Original work published 1962)

Levitt, H., Motulsky, S., Wertz, F., Morrow, S., \& Ponterotto, J. (2017). Recommendations for designing and reviewing qualitative literature in psychology: Promoting methodological integrity. Qualitative Psychology, 4(1), 2-22.

Levitt, J., Bamberg, M., Creswell, J., Frost, D., Josselson, R., \& Suarez-Orozco, C. (2018). Journal article reporting standards for qualitative primary, qualitative metaanalytic, and mixed methods research in psychology: The APA publications and communications board task force report. American Psychologist, 73(1), 26-46.

Marich, J. (2010). EMDR in addiction continuing care: A phenomenological study of women in early recovery. Psychology of Addictive Behaviors, 24(3), 498-507.

Marich, J. (2012). What makes a good EMDR therapist?: Exploratory clients from client-centered inquiry. Journal of Humanistic Psychology, 52(4), 401-422.

Marsden, Z., Lovell, K., Blore, D., Ali, S., \& Delgadillo, J. (2017). A randomized controlled trial comparing EMDR and CBT for obsessive-compulsive disorder. Clinical Psychology \& Psychotherapy. 25(1): e10-e18. https: / / doi.org/ / 10.1002/cpp.2120

Moustakas, C. (1994). Phenomenological research methods. Thousand Oaks, CA: Sage Publications.

McCracken, G. (1988). The long interview: Qualitative research methods, volume 13. Newbury Park, CA: Sage.

Morse, J. M., Barrett, M., Mayan, M., Olson, K., \& Spiers, J. (2000). Verification strategies for establishing reliability and validity in qualitative research. International Journal of Qualitative Methods, 1(2), 1-19.

Reisetter, M., Korcuska, J. S., Yexley, M., Bonds, D., Nickels, H., \& McHenry, W. (2004). Counselor educators and qualitative research: Affirming a research identity. Counselor Education and Supervision, 44, 2-16.

Ricci, R. J., \& Clayton, C. A. (2008). Trauma resolution treatment as an adjunct to standard treatment for child molesters. Journal of EMDR Practice and Research, 2(1), 41-50.
Ricci, R. J., Clayton, C. A., \& Shapiro, F. (2006). Some effects of EMDR on previously abused child molesters: Theoretical reviews and preliminary findings. Journal of Forensic Psychiatry and Psychology, 17, 538-562.

Saldaña, J. (2015). The coding manual for qualitative researchers. Washington, DC: Sage.

Shapiro, F. (2018). Eye movement desensitization and reprocessing therapy: Basic principles, protocols, and procedures (3rd ed.). New York, NY: The Guilford Press.

Thomson, S. B. (2011). Sample size in grounded theory. Qualitative Research Validity: JOAAG, 6(1), 77-82.

Tucker, J.A., Donovan, D.M., \& Marlatt, G.A. (1999). Changing addictive behavior: Bridging clinical and public health strategies. New York: The Guilford Press.

Van Toorenburg, M., Sanches, S., Linders, B., Rozendaal, E., Voorendonk, A., Van Minnen, A., ... De Jongh, A. (2020). Do emotion regulation difficulties affect outcome of intensive trauma-focused treatment of patients with severe PTSD? European Journal of Psychotraumatology, 11(1): 1724417 https: / / doi.org/10.1080/20008198.2020.1724417

Whitehouse, J. (in press). What do clients say about their experiences of EMDR in the research literature? A systematic review and thematic synthesis of qualitative papers. European Journal of Trauma and Dissociation. 3(1), 17-21 https: / / doi.org/10.1016/j.ejtd.2019.03.002

Willig, C. (2013). Introducing qualitative research in psychology: Adventures in theory and method. Buckingham, UK: Open University Press.

Wise, A., \& Marich, J. (2016). The perceived effects of standard and addiction-specific EMDR therapy protocols. Journal of EMDR Practice and Research, 10(4), 231-244.

World Health Organization. (2013). Assessment and management of conditions specifically related to stress $m h$ GAP Intervention Guide Module. Geneva, Switzerland: Author. Retrieved from http://apps.who.int/iris/bitstream/ 10665 / 85623 / 1/9789241505932_eng.pdf?ua =1

Yin, R. K. (2003). Case study research: Design and methods (3rd ed.). Thousand Oaks, CA: Sage.

Disclosure. The authors have no relevant financial interest or affiliations with any commercial interests related to the subjects discussed within this article.

Funding. The author(s) received no specific grant or financial support for the research, authorship, and/or publication of this article.

Correspondence regarding this article should be directed to Jamie Marich, The Institute for Creative Mindfulness, P.O. Box 8732, Warren, OH 44484. E-mail: jamie@ jamiemarich.com 